\title{
Effectiveness of Routing Protocols for Different Networking Scenarios
}

\author{
Akbor Aziz Susom* \\ Institute of Information and Communication Technology, Bangladesh University of Engineering and Technology, 1205, Bangladesh.
}

\section{A R T I C L E I N F O}

Article history:

Received: 01 June, 2018

Accepted: 26 June, 2018

Online: 23 July, 2018

Keywords:

Routing Protocols

Hybrid Networks

Throughput

Jitter

Packet Length

Packet Loss

\begin{abstract}
A B S T R A C T
Selection of a routing protocol is vital for modern arena of Internet communication as network traffic and network complexities are rapidly increasing. This paper evaluates the effectiveness of three routing protocols namely routing information protocol version 2 (RIPv2), open shortest path first (OSPF), enhanced interior gateway routing protocol (EIGRP), and hybrid protocols based on these three. These stand-alone and hybrid protocols are compared in terms of four metrics: throughput, jitter, packet length and packet loss. Different kinds of networks are developed using Graphical Network Simulator3 (GNS3) network software emulator. The network performance measurement of those four metrics is done with the help of Wireshark and Iperf tools. Besides, three network topologies termed as 'experimented', 'retracted' and 'extended' networks are created using 7, 3, and 9 routers. Simulation results indicate that EIGRP protocol performs the best showing the highest average throughput (28 packet/sec), while RIP-OSPF hybrid protocol has the lowest average throughput (16 packet/sec). Moreover, EIGRP has the lowest value of packet loss of 2.66. The lowest jitter value is obtained for hybrid RIP-OSPF-EIGRP protocols. However, EIGRP has a moderately high jitter value. Furthermore, EIGRP has standard size of packet length. Hence, EIGRP can be a good selection as a routing protocol for different sized networks.
\end{abstract}

\section{Introduction}

Connection of group of routers that are used to build different networking systems based on the requirements and affordability mainly follow two functions - select a path through networks and then transmit information packet across that fixed path to reach the determined destination. In doing so, [1] routing protocols and algorithms play most significant role to plot the routes through networks so that packet can transmit to the most efficient possible paths. First routing protocol shares information among immediate neighbors, and then throughout the network [2]. This way, routers gain information of the topology of the network. Throughout this process, routing protocols follow some criteria based on throughput, delay, efficiency, simplicity, low overhead, reliability/stability, and flexibility [3]. Therefore, performance and efficiency of a networking system is dependent on the routing protocols. As it varies protocol to protocol as well as performance changes in different networking systems. In this experiment, the purpose is to find throughput, jitter, Packet length and packet loss

*Akbor Aziz Susom, Bangladesh University of Engineering and Technology Email: akb.susom@gmail.com

www.astesj.com

https://dx.doi.org/10.25046/aj030412 in three different networks with seven different combinations of protocols. Then to compare those results with each other from protocols to protocols, and system to system.

As with most complex technologies and requirements for diversity, there's no one-size-fits-all solution when it comes to develop networking systems [5]. The necessity and resources of each unique sector will correlate to a different set of networking systems and solutions. [6] Therefore, development and changes in the development of the networking systems has been a vital need for fulfilling different demands and requirements. It is needed to carefully consider the situation and determine to change the network design for their situation to create an optimized networking solution for that situation [7]. For that reason, in this research paper we have analyzed with three different networks on the basis of experimented networks that uses 7 routers then we extended and retracted the system by corresponding 9 and 3 routers respectively to diversify the networking systems.

A network that has been well designed is characterized by consistency and performance of some parameters and protocols used in the networks. A consistently high level of performance is 
observed with the good combination of different networking protocols [8]. As Routing protocols has significant influence on networking system also each routing protocols have both positive sides and negatives sides on different networking systems.

So, we have selected three routing protocols-RIP, OSPF, EIGRP and the hybrid protocols consisting of these three individual ones. [9] An important point to note that all through this study, RIPv2 is considered as RIP. The hybrid protocols considered are RIPOSPF, RIP-EIGRP, OSPF-EIGRP and RIP-OSPF-EIGRP. It can be noted that the three individual protocols: RIP (version 2), OSPF and EIGRP are supported by VLSM, and these have built in algorithms of Bellman-Ford, Dijkstra and Dual, respectively [10].

These protocols are used for evaluating not any single networking system rather focusing on different networking systems to find the effective and efficient match of routing protocols and algorithms for that specific system [11] and also to suggest whether it satisfies the need of design or redesign of different networking infrastructure based on some parameters like throughput, Jitter, Packet Length and Packet Loss.

This research is determined to do a comparative analysis of routing protocols alone and combined routing protocols performance in different networks [12]. Finally, to suggest the best combination of routing protocols that will meet the requirements of the networking system as well as to show the best performance for the particular computer networks.

The rest of the paper is organized as follows. Section 2 presents the methodology of the study, Section 3 describes the related work, and Section 4 presents the performance results obtained from computer simulations. Finally, Section 5 provides the concluding remarks and future research directions.

\section{Methodology}

\subsection{Procedures}

With seven combination of routing protocols in three different networks, first to establish twenty-one different networks with specific router configuration that are all configured with real time topology. This research then executed to create traffic with transmission of information packet over a fixed time period to observe the real time figure and values of different performance measuring parameters of the networks and analyzing the captured traffic based on these parameters. This paper first measured each protocols throughput and jitter value both in software and manually with raw data for every networks using the same resources and conditions. Showing each protocols graph for both throughput and jitter value is demonstrated in section 4 (simulation results portion of the paper). Similarly packet length value and packet loss percentage are measured with the same conditions of the networks. Finally, to demonstrate the comparison graph for each routing protocols in three different networks provide the significant ideas of the protocols performance and efficiency. Depending on the comparison graph, decision has been made in the conclusion section to determine the best combination of routing protocols that can be implemented in a particular networking system.

\subsection{Software and Simulation Tools}

The Network has been established in Graphical Network Simulator 3 (GNS3) version 2.1.5 using VirtualBox version 5.2.12 that runs on the Windows 10 operating system. The Router 3725 Series with Cisco IOS operating system enabled that runs in GNS3, and Windows 10 operating system is running in VirtualBox as a PC. For simulation purpose, Wireshark software version 2.5.1 is used and version 3.1.3 Iperf applications running on the PC.

\subsection{Prototype Modeling}

Simulation will be performed in three different networks named as Experimented, Extended and Retracted Networks with a combination of routing protocols in seven different scenarios following as RIP, OSPF, EIGRP, RIP-OSPF, RIP-EIGRP, OSPFEIGRP, RIP-OSPF-EIGRP.

\subsection{System Design}

We consider three different networking models - retracted network, experimented network and extended network, for this experiment. The models have been developed on the basis of real time topology with combination of 3, 7 and 9 routers respectively. Two computers are also connected with the networks for the transmission of the packet to create traffic that is used for simulation purpose of the network [13]. GNS3 software has been used to design the network that is as followed:

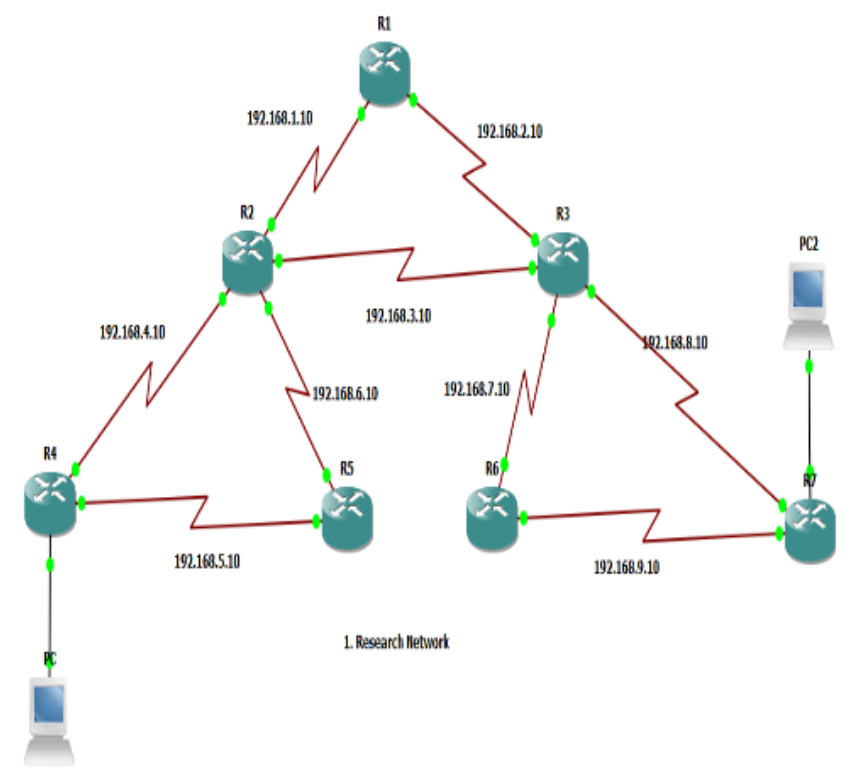

Figure 1: Experimented Network

\subsection{Parameters to evaluate the protocols}

\section{A. Throughput:}

Network throughput refers to the average data rate of successful communication of a network or message delivery over a specific networking link. [11] It measures a comparable effectiveness and efficiency of an operation or a system. It defines how strong and consistent the connection is maintained during the session. It is calculated by a theory as: 
Throughput for each connection(i)

$$
=\sum \frac{(\text { Throughput (i)) }}{\sum_{\mathrm{J}-1}^{\mathrm{N}} \quad \text { Throughput (j) }}
$$

In our experiment, we have measured throughput value for each system to find the throughput for that specific protocol or combined protocols to see the transmission of packet over that fixed period in a network.

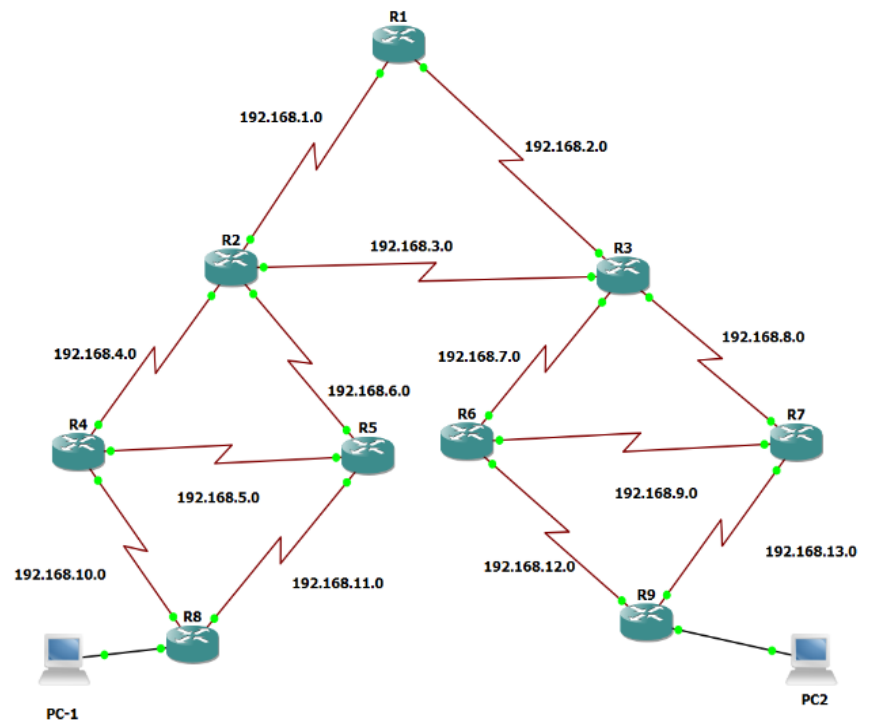

Figure 2: Extended Network

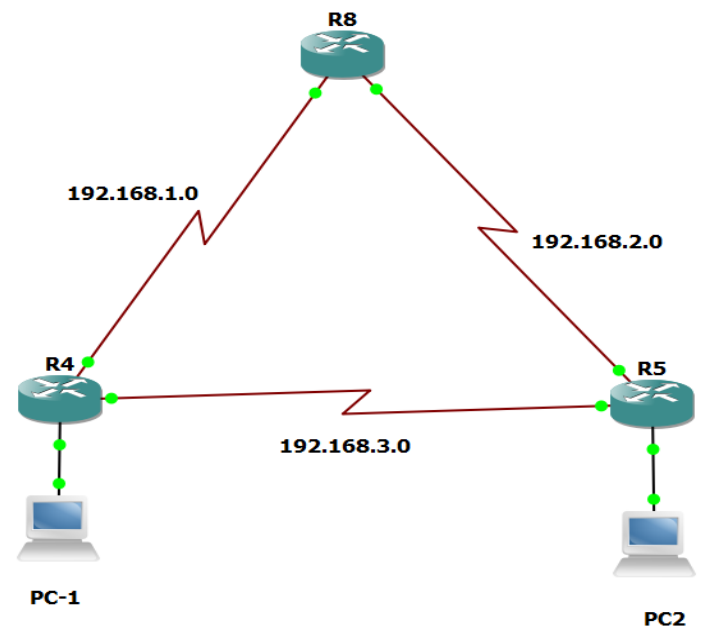

Figure 3: Retracted Network

\section{B. Jitter}

Jitter refers to statistical variation of Packet Delay and it is cited in IETF RFC 3393 and 5481.It is calculated to find the delay for all the packets from a source to a destination. Here, we have both calculated the jitter value in iperf software and taking the raw data from Wireshark that is calculated as follows:

Jitter rate $=$ Total Variant Delay $/$ No. of packet sent -1

Measuring the total delay,
Total Variant Delay $=(\mathrm{Ri}-\mathrm{Si})(\mathrm{R} \mathrm{i}+1-\mathrm{Sj}+1)$

Here, $\mathrm{Ri}=$ Received Time

$\mathrm{Si}=$ Sent Start time

\section{Packet Length}

Packet size is a considerable issue for energy constrained and performance evaluation of a network. Because of larger size of packets, data bit corruption creating higher frequency of retransmission may be caused. And bigger packet might have problem if it is above the size of MTU (Maximum Transmission Unit). Moreover, NIC (Network Interface Controller) and OS has memory size restriction [14]. On the other hand, small size packets are more efficient but creating too short packet size might cause faults, like higher overhead and startup energy consumption for each packet can degrade the network performance. Besides, small size packets have issues with fragmentation and security problem. For this reason, Packet length for protocols and combination of the protocols in three different networks is measured to evaluate the particular networking system.

\section{Packet Loss}

Packet loss refers to small bits of lost data over a transmission period to or from one networking equipment to another equipment. Some amount of packet loss, generally just a small percentage can be available in a connection of a network. However, Packet loss is closely associated with quality of service considerations as network performance is impacted by packet loss and retransmission. Because of saturation, bandwidth outage, misconfiguration, network may be defected to lose acknowledge when retransmission is needed although the packet was transmitted. Moreover, TCP congestion window size is affected for packet loss when it will not accept optimal throughput value for the network.

\section{Related works}

There are several papers related to evaluation of networking protocols performance like dynamic routing protocols- RIP, OSPF and EIGRP and using these protocols to conduct the other scientific research. In paper [2] author describes the routing protocols using same Opnet software while they considered point to point throughput, querying delay and convergence time to compare the protocols. They both suggested EIGRP protocols for best choice In paper [4] they analysis performance of Virtual private Lan service network using Kerberos-enabled protocols (alternative authentication protocols) to measure the throughput value with respect to Normal VPLS network using Wireshark software IO graph. However, some other parameters like Delay, time factor, transmission efficiency is also important to precisely measure the performance of an authentication protocols in VPLS network. Paper [5] evaluated the routing protocols while they also considered combined routing protocols performance in Ipv6 network using iperf software which measured the throughput, jitter and packet loss value in a same networks platform. After all this result may change with the demand or design of different other computer networks. In the paper [6], author examined the performance of Ipv4 and Ipv6 when routing protocols have been utilized in both Ipv4 and Ipv6 virtual networks using GNS3 
simulator software. They compared end to end delay and latency result for Ipv4 and Ipv6 and commented Ipv6 is the better choice for these two parameters. In paper [10] author analysis of RIP, OSPF and EIGRP protocols performance using Opnet software where they considered network convergence, Average Delay, Average email uploads response time, protocols traffic etc. to compare the performance.

But they have performed all these simulation experiment in fixed network structure that results from each simulation might not be the same when network design changes. Besides, Paper [ 11] compared the routing protocols performance in Big data application using Riverbed Modeller Simulator while they made comparison based on convergence duration, video conferencing packet end-to-end delay, jitter of voice and voice packet end-toend delay.

\section{Simulation Results:}

\subsection{Throughput}

This experiment has been done to measure the throughput value in terms of different protocols and hybrid protocols for three types of networks. Here, $\mathrm{x}$ and $\mathrm{y}$ axis are considered as time and average packet sent over that time period. The average throughput value is calculated as packet per second. For the simulation purpose, SMA period is set as 10 interval SMA and corresponding value for $y$ axis is considered as 1000 interval SMA. Specific protocol is selected every time to find the throughput value for the graph.

\section{For Experimented Network:}

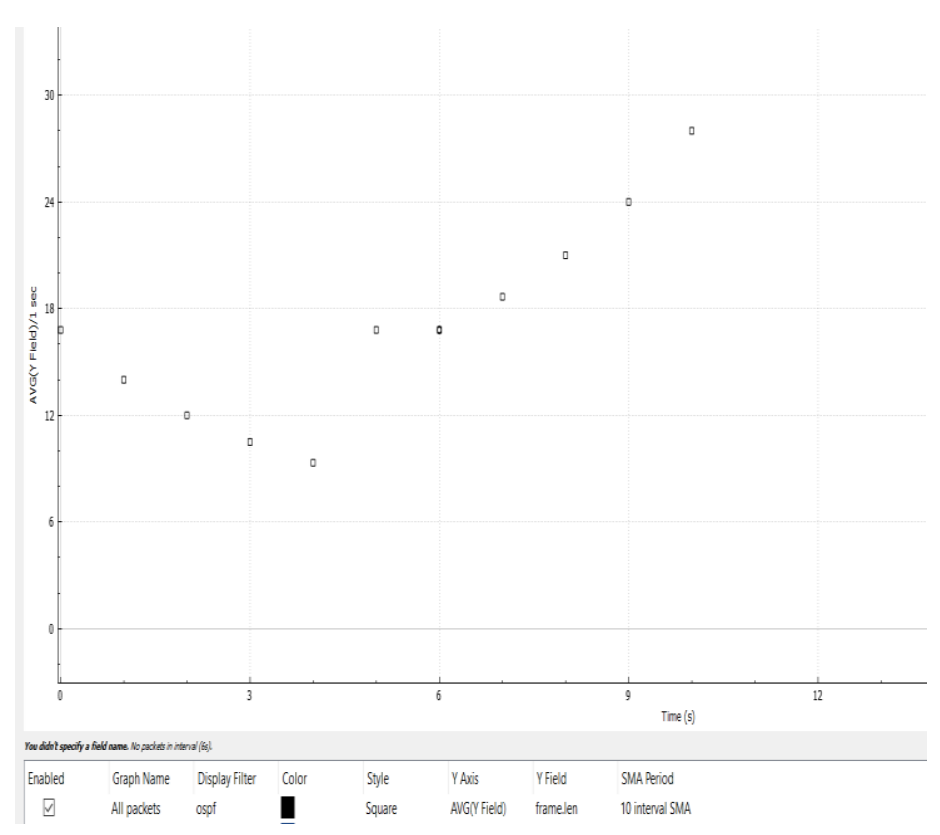

Figure 4: Average Packet / sec as throughput vs time for OSPF

In this figure, we have found that the average throughput value for this network using ospf protocol is 18 packet / sec which show maximum throughput is 28 . The value of throughput initially got down up to 5 secs then went upward.

\section{For extended Network:}

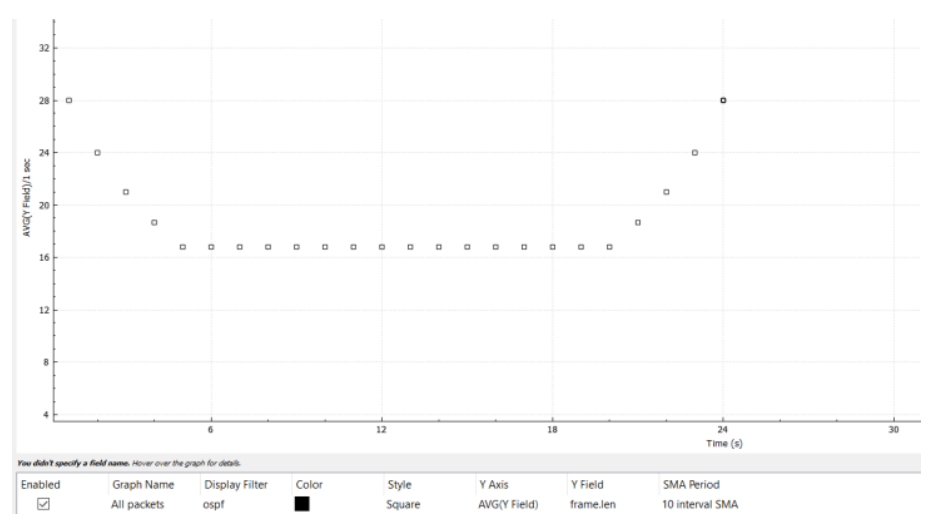

Figure 5: Average Packet / sec as throughput vs time for OSPF

In this figure, the average throughput value for this network using ospf protocol is 17 packet / sec which show maximum throughput is 26 .

\section{For Retracted Network:}

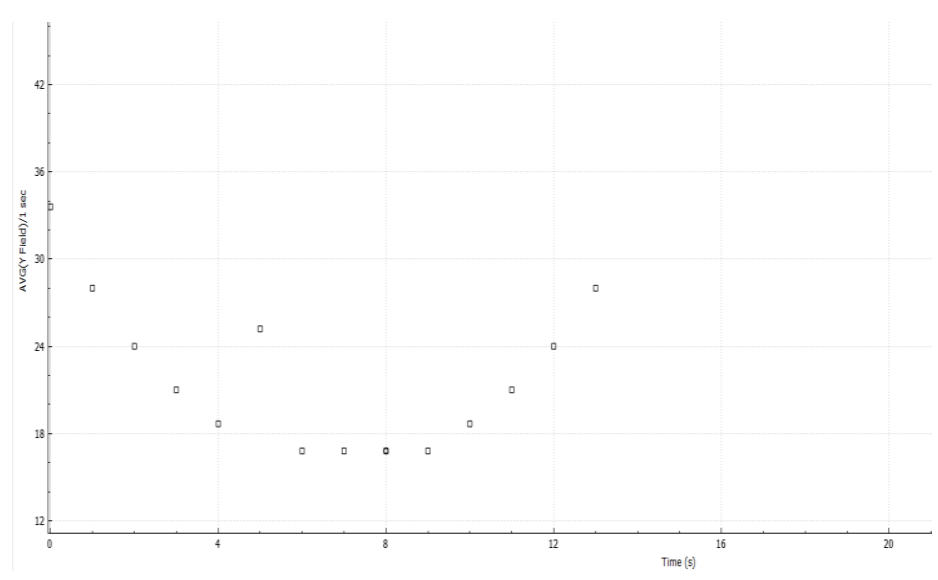

Figure 6: Average Packet / sec as throughput vs time for OSPF

In this figure, the average throughput value for this network using ospf protocol is 20 packet / sec which show maximum throughput is 34. Above the simulation figure has been shown for OSPF protocols in three different networks. The similar way is followed for valuation of other protocols to finally demonstrate the comparison graph for standalone and hybrid protocols.

\section{Valuation:}

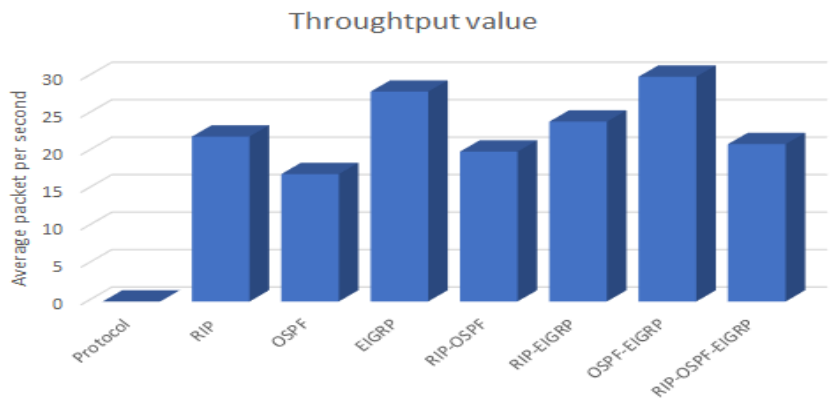

Figure 7: Comparison graph of Protocols vs average packet per second as Throughput for Extended Network. 
Thrpughtput value for Extended Network

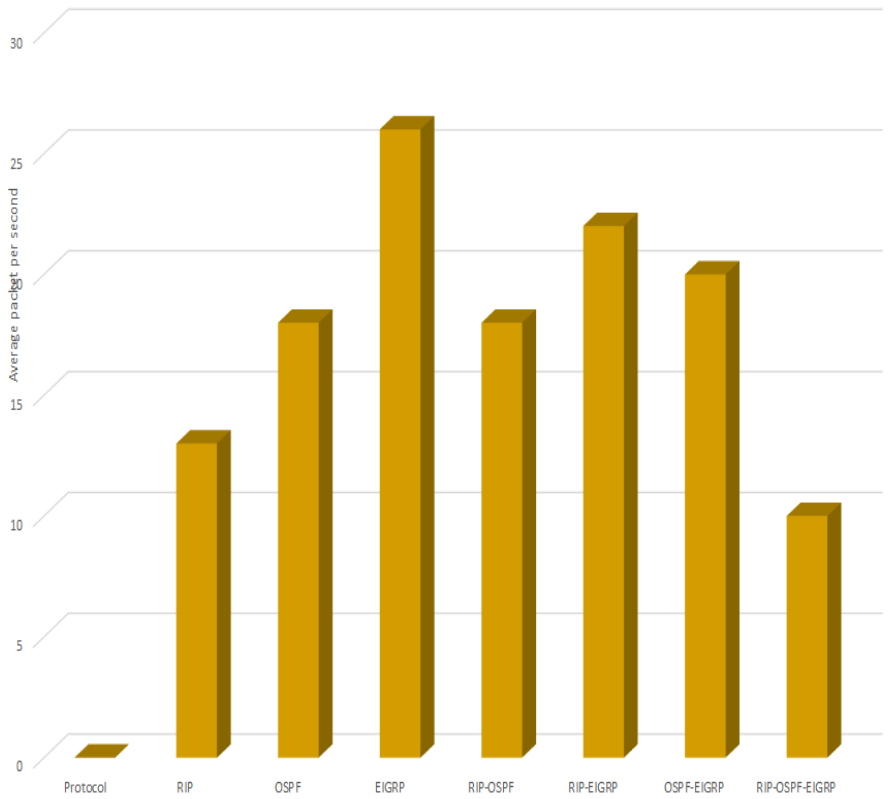

Figure 8 Comparison graph of Protocols vs average packet per second as Throughput for Extended Network.

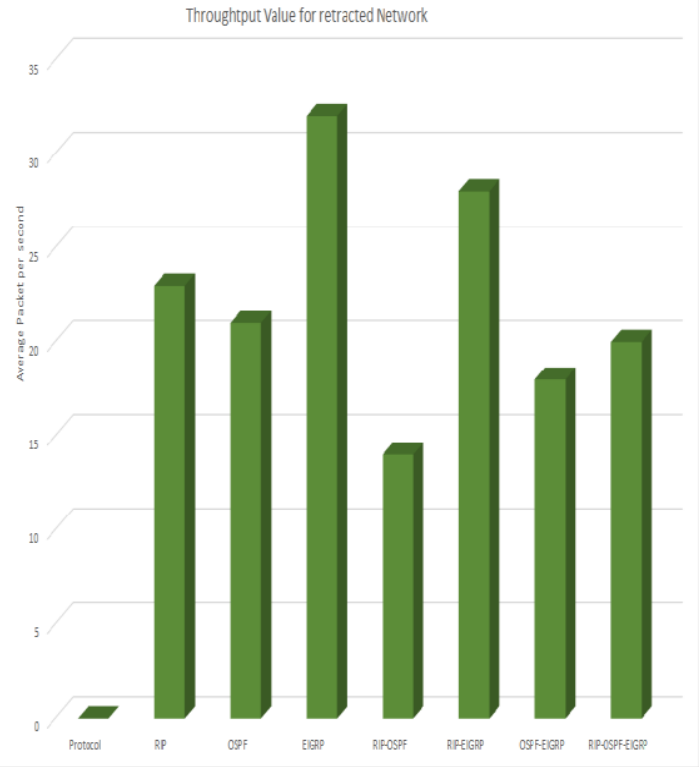

Figure 9: Comparison graph of Protocols vs average packet per second as Throughput for Retracted Network.

\section{Results Analysis and decision:}

Analyzing the above figures, combined OSPF-EIGRP protocol performs better for experimented network with maintaining average throughput almost 30 packets per sec. Here alone EIGRP and combined RIP-EIGRP maintains average throughput 28 and 23 respectively. Poor performance is observed for OSPF and RIPOSPF protocols that are below throughput value of 20 .

Extending the network, best performance is obtained for alone EIGRP (above 25 packets per second) which was actually proposed by researchers for big networking system. Lower
\& performance is shown for combination of three protocols and RIP protocols that is below 13 packets per second. Similarly, EIGRP protocol achievement for retracted network is also the highest number of throughput value that is 32 packets per second while RIP-EIGRP and alone RIP protocols have shown considerable performance for this networking system. However, least throughput is attained for combined RIP-OSPF protocols.

Performance gain is obtained for RIP and EIGRP protocols for Retraced Network compared to Experimented network that are $9.52 \%$ and $23.07 \%$ while performance penalty is observed for combined RIP-OSPF protocols $(46.15 \%)$.

Compared to extended network with experimented network, Performance penalty is obtained for combined OSPF -EIGRP $(27.27 \%)$ which was the suggested for experimented network.

\subsection{Jitter}

In this simulation process, jitter value is calculated maintaining seven different scenarios in three different networks. It shows the deviation during the transmission of the packets as shown in $\mathrm{x}$ axis with respect to time in y axis for every particular networking system.

Jitter value is calculated in both ways by iperf application and manually to take the raw data from capture data in each networking module for specific protocols to find the actual figure and then to compare those to select the best one.

Below the simulation figure for jitter has been shown for OSPF protocols in three different networks. The similar way is followed for valuation of other protocols to finally demonstrate the comparison graph for standalone and hybrid protocols.

\section{For Experimented Network:}

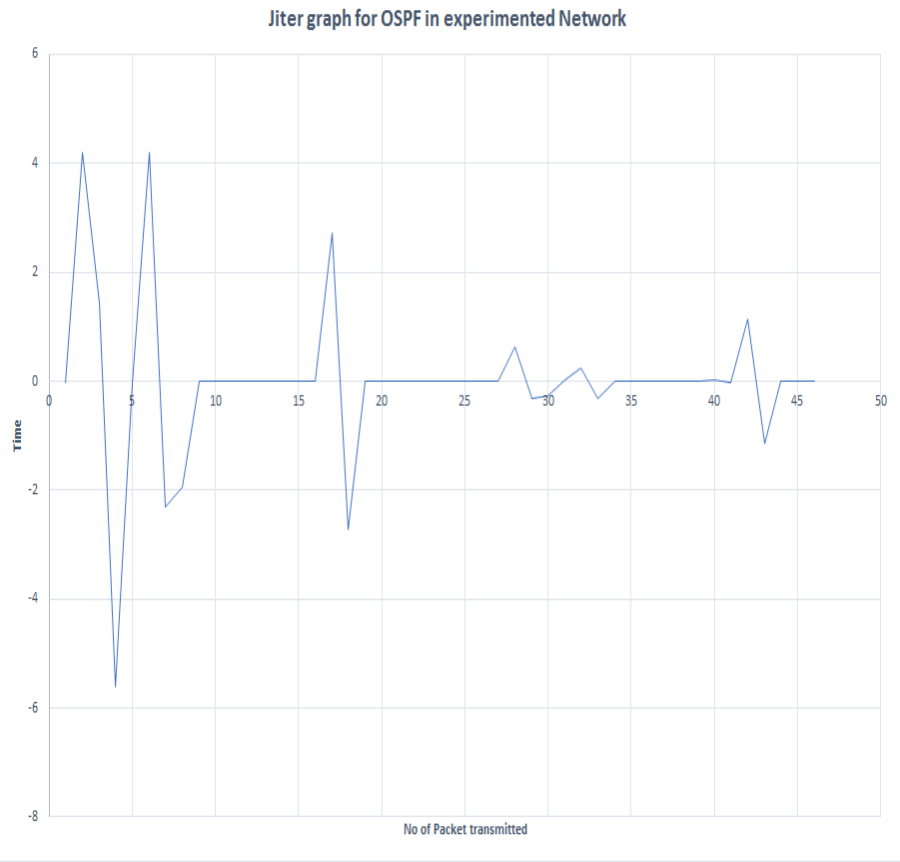

Figure 10: Variation of time as Jitter graph for OSPF 


\section{For Extended Network:}

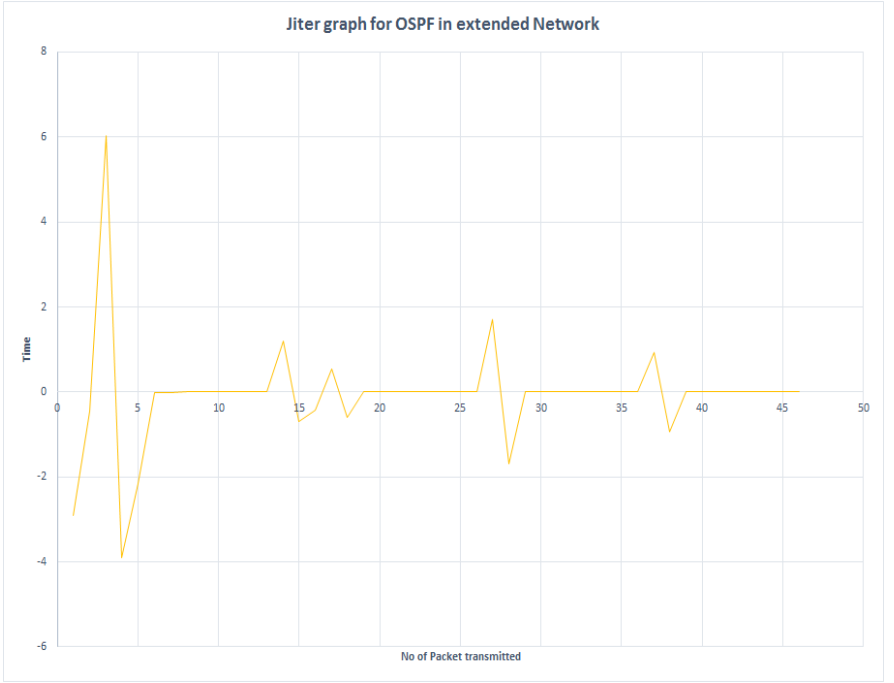

Figure 11: Variation of time as Jitter graph for OSPF

\section{For Retracted Network:}

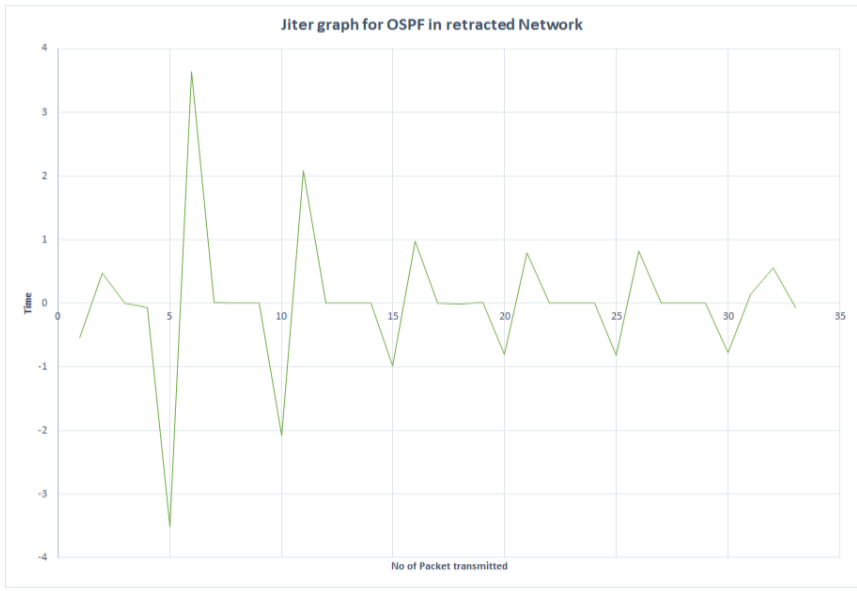

Figure 12: Variation of time as Jitter graph for OSPF

\section{Valuation:}

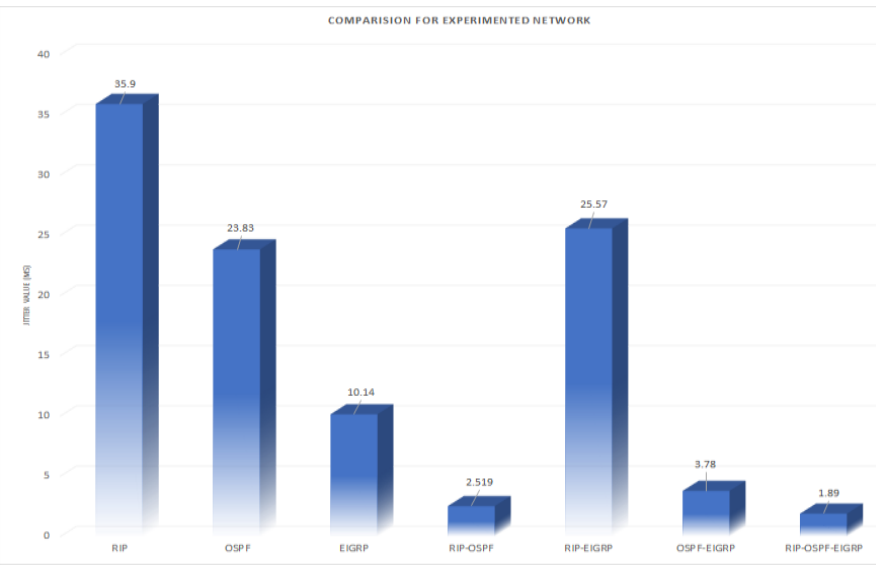

Figure 13: Comparison of Jitter Value in ms through y axis for Experimented Network

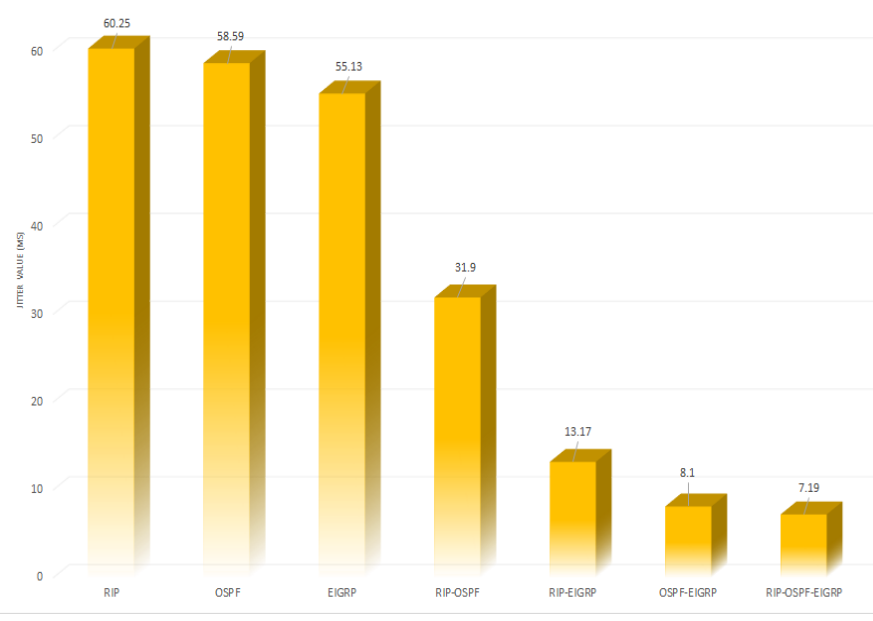

Figure 14: Comparison of Jitter Value in ms through y axis for Extended Network

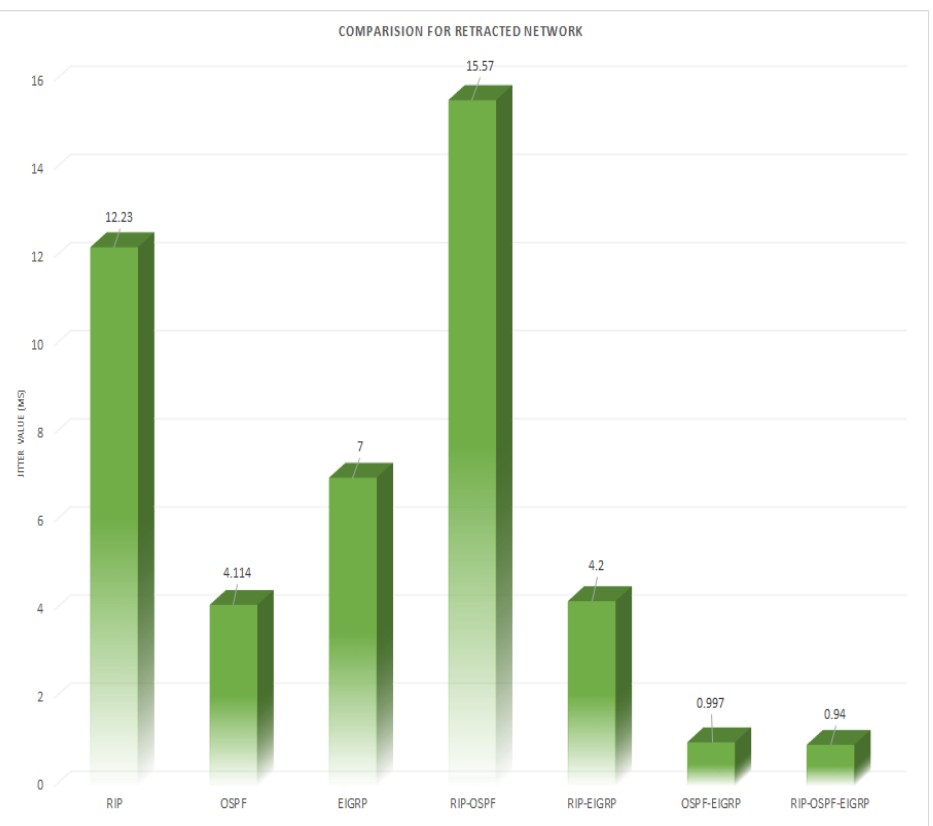

Figure 15: Comparison of Jitter Value in ms through y axis for Retracted Network

\section{Result analysis and decision:}

Minimum jitter value captured for the protocols is considered the best one for that particular networking system. Following the experimented network, the lowest jitter value is got for combination of three protocols in a network "RIP-OSPF-EIGRP" that is $1.89 \mathrm{~ms}$. RIP-OSPF and OSPF-EIGRP also show the good result for jitter are 2.5 and 3.78 respectively. The very poor result observed for the protocol of RIP (35.9ms). Similar result is also obtained for retracted and extended network considering the flow graph performance of the protocols that RIP-OSPF -EIGRP combined networking protocols show the best performance for every networking design. However, for retracted network, performance increased for every combination and alone protocols comparing the jitter value. Considerable performance shown for 
RIP, OSPF, RIP-EIGRP that are gained by $65.93 \%, 81 \%$ and $83.57 \%$ respectively for retracted network. Only combine RIPOSPF performance got down as jitter value increased from 2.51 to 15.57 in Retracted network.

\subsection{Packet Length}

Packet Length is measured considering ten different range of the size of the packet that is described through $\mathrm{x}$ axis and $\mathrm{y}-$ axis is cited as the number of packet in a particular range in percentage. The result is obtained first for each protocol and combined protocols then showing the comparison graph on the basis of the simulation results. Below the table for packet length has been shown for OSPF protocols for three different networks. The similar way is followed for valuation of other protocols to finally demonstrate the comparison graph for standalone and hybrid protocols.

\section{Scenario 1.}

Packet Length graph for OSPF

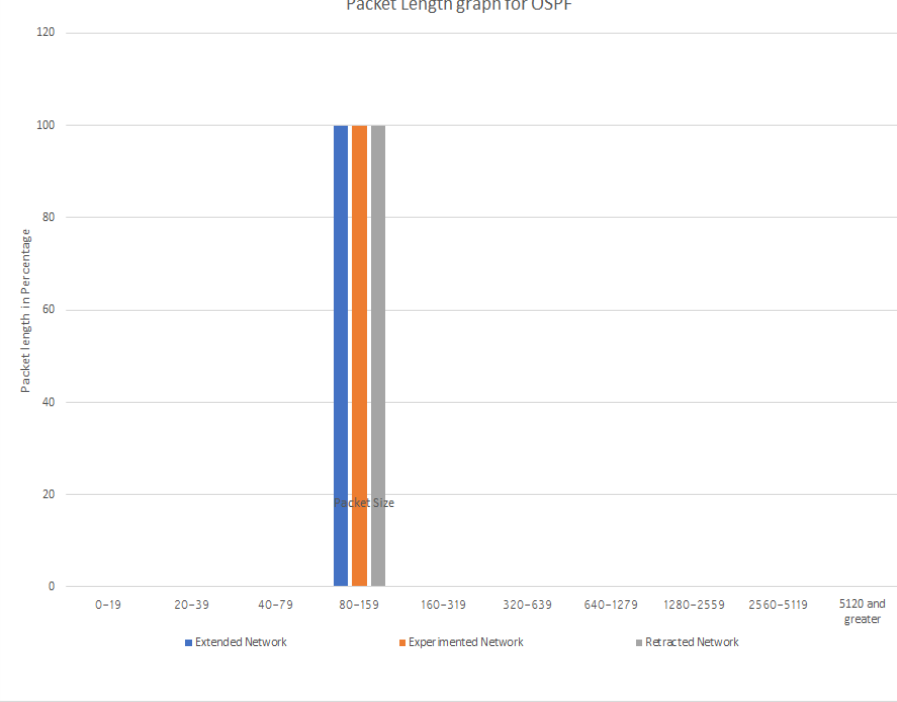

Figure 16: Packet Size for OSPF.

Valuation:

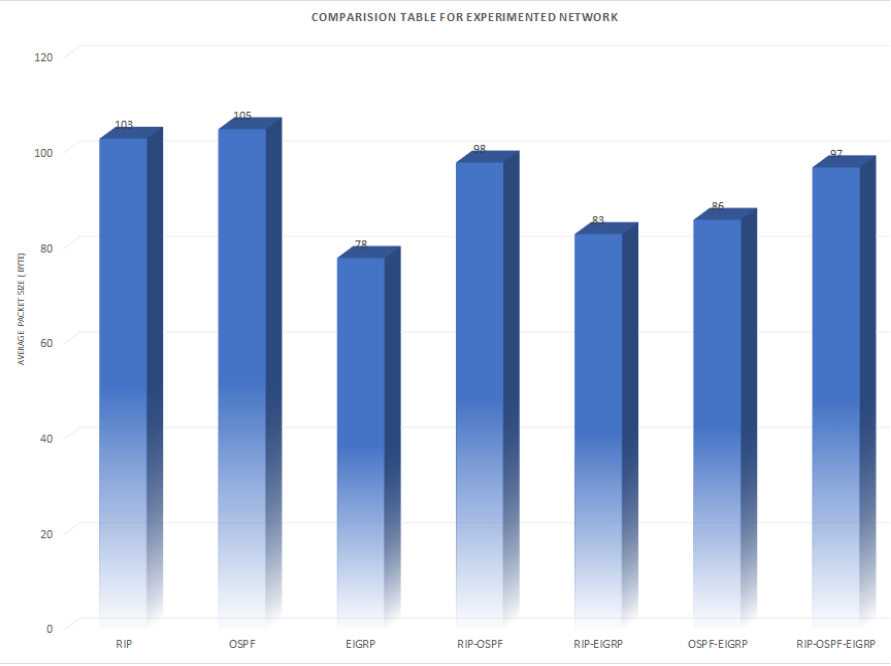

Figure 17: Comparison for protocols vs average packet size in byte for Experimented Network.

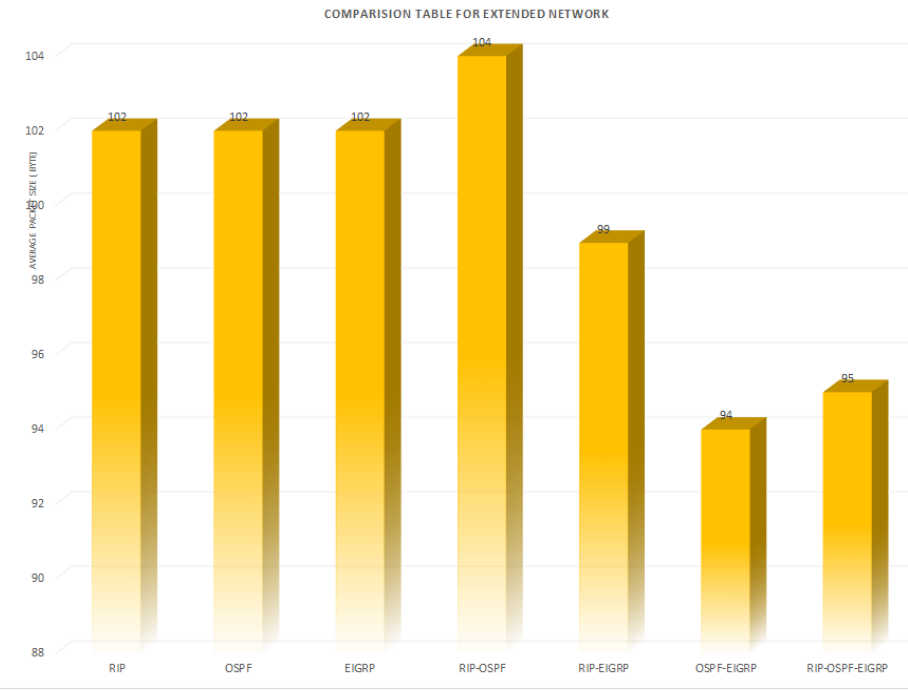

Figure 18: Comparison for protocols vs average packet size in byte for Extended Network.

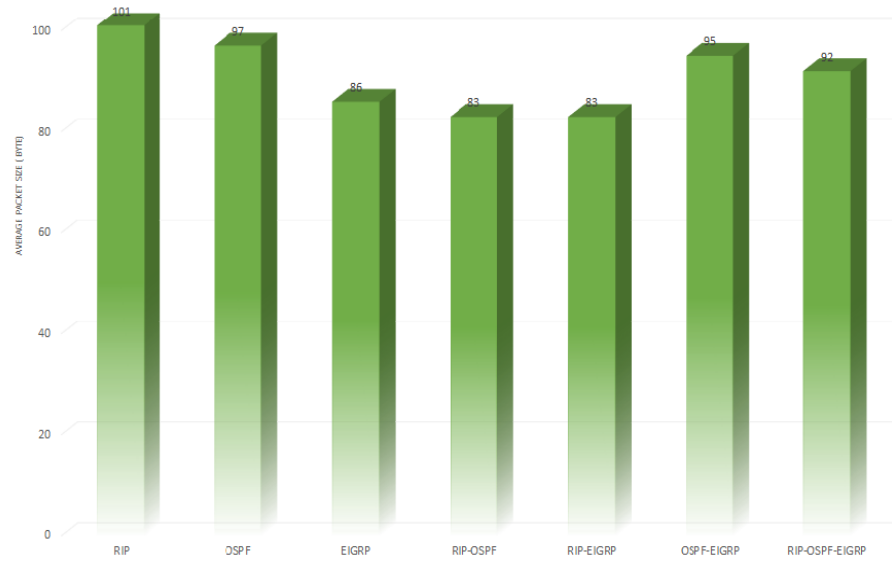

Figure 19: Comparison for protocols vs average packet size in byte for Retracted Network.

\section{Result Analysis and Decision:}

There is no big difference found in packet size during the transmission of the packet in all there networking system. For experimented network, packet size ranges from 78 to 106 for protocols while highest average packet size got for OSPF is 106 and lowest size for EIGRP is 78. In the case of extended network, it ranges from 94 to 104 while considerable expansion of average packet size captured for RIP-OSPF is 104 that was 83 for experimented network. No similar size packet is seen for extended network compared to retracted network.

On the contrary, average packet size for retracted network ranges from 83 to 101 which shows minor difference in packet length for all protocols. Here, same average packet size is obtained for RIPOSPF and RIP-EIGRP, is 83. The maximum packet size is seen for RIP is 101 that is somewhat similar to all other networking systems. Overall, RIP and OSPF protocols show bit higher packet size for the network. 


\subsection{Packet Loss Comparison Graph}

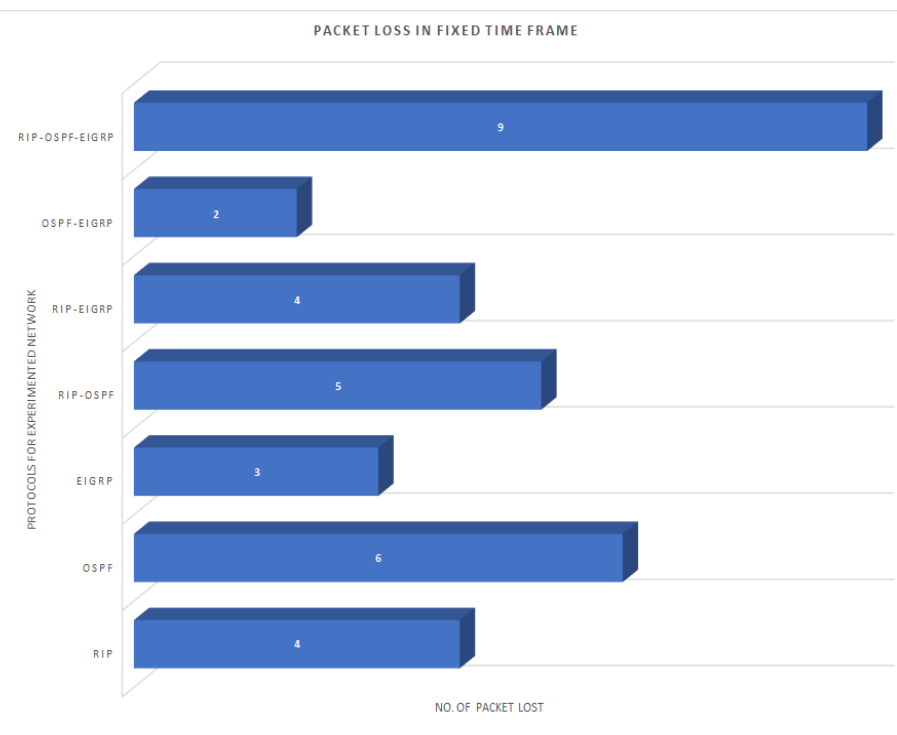

Figure 20: Protocols vs No of Packet loss in Experimented Network.

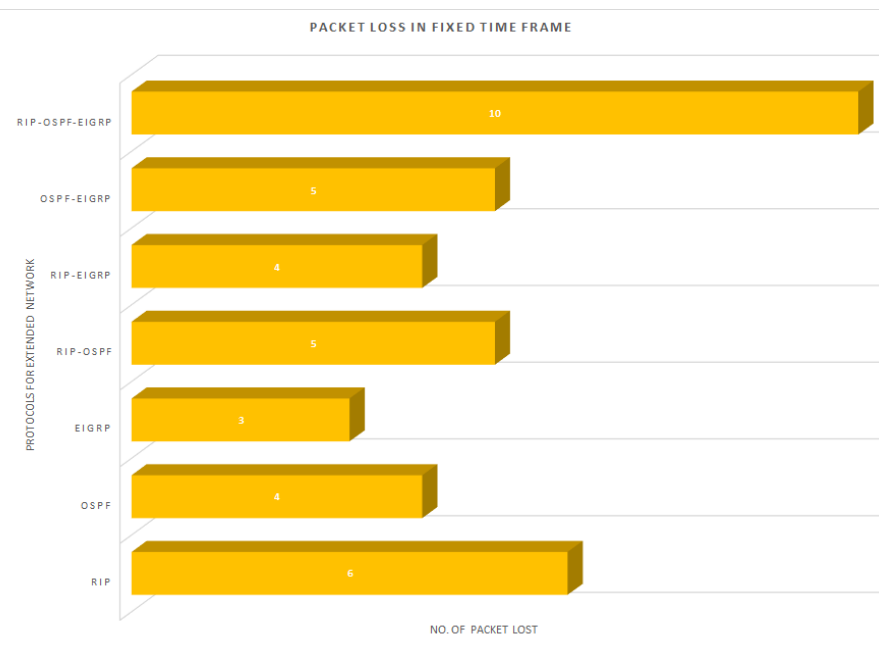

Figure 21 Protocols vs No of Packet loss in Extended Network.

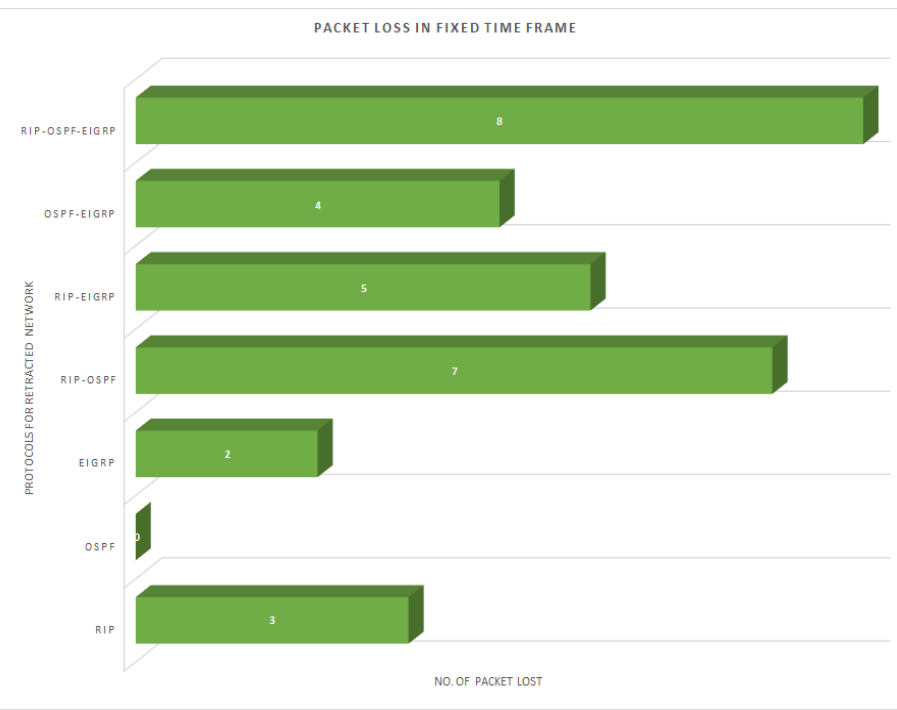

Figure 22: Protocols vs No of Packet loss in Retracted Network.

\section{Result Analysis and Decision:}

Comparison graph for experimented network indicated that the average percentage of the packet loss is lowest for OSPF-EIGRP as it shows packet loss value is 2 during the transmission steam of the packet. The second-best performance shown for this network is EIGRP having packet loss value 3. However, poor performance is shown for RIP-OSPF-EIGRP and OSPF for this network that shows 6 and 9 packet loss value during the transmission of the packet.

The performance is still satisfactory for EIGRP in extended network while OSPF and RIP-EIGRP show the same value of packet loss in this network. Combination of three protocols show the maximum packet loss value in extended network is 10 while it was 9 for experimented network. In the case of retracted network, excellent result shown by OSPF having zero packet loss while RIP-OSPF and RIP-OSPF-EIGRP depicts poor performance as usual in packet loss for all networking systems. Finding the reason for this result for these two protocols, raw data shows many number of retransmission request and drop packet that causes higher packet loss percentage for RIP-OSPF and RIPOSPF-EIGRP.

\section{Conclusion and Future works:}

In this paper, we designed and evaluated Routing protocols namely RIP, OSPF, EIGRP along with its all possible combinations for three different networks and applied it to measure throughput, jitter, packet length and packet loss to demonstrate its performance and utility. We demonstrated how the results obtained in one network design can change for the configuration of the same protocol in response to dynamically changing conditions of another network. On comparing the results of the simulation of different protocols and combined protocols, the overall best performance is shown for throughput value by EIGRP protocols while only for experimented network, OSPFEIGRP combined protocol is suggested to implement that give the highest throughput. In fact, RIP-EIGRP and OSPF-EIGRP can be chosen as the second-best selection for the networks. The average throughput considering all networks for EIGRP is 28.00 packet/sec while the lowest obtained by combined RIP-OSPFEIGRP average 14.00 packet/sec respectively. After that, the suggestion goes to combined protocols of either RIP-OSPFEIGRP or OSPF-EIGRP in term of jitter value, that shows minimum packet delay in all networks conditions. The average minimum jitter value for RIP-OSPF-EIGRP and OSPF-EIGRP are $3.34 \mathrm{~ms}$ and $4.29 \mathrm{~ms}$ respectively while the poor performance having maximum jitter is examined for RIP is average $36.12 \mathrm{~ms}$ meaning all three networks. Moreover, mixed results are captured for packet length in different networks. In general, RIP, OSPF and RIP-OSPF shows the standard and largest packet size during the networks communication. Finally, for the best possible value for packet loss is obtained by EIGRP which average packet loss value is 2.66 in overall all three networks whereas RIP-OSPF has overall 5.66 packet loss. The results backed by simulation evaluation and validation demonstrate that EIGRP and combined OSPF-EIGRP are best solution to choose routing protocols for enhancing networking performance especially for extended (large-scale) networks while RIP or OSPF can have moderate performance for retracted (small-scale) network. 


\begin{tabular}{|c|c|c|c|c|c|c|c|c|c|c|c|c|c|c|c|c|}
\hline \multirow{2}{*}{ Protocols } & \multicolumn{4}{|c|}{ Experimented Network } & \multicolumn{4}{|c|}{ Extended Network } & \multicolumn{4}{|c|}{ Retracted Network } & \multicolumn{4}{|c|}{ Average } \\
\hline & $\begin{array}{l}\text { Throu } \\
\text { ghput }\end{array}$ & Jitter & $\begin{array}{l}\text { Packet } \\
\text { Length }\end{array}$ & $\begin{array}{l}\text { Packet } \\
\text { Loss }\end{array}$ & $\begin{array}{l}\text { Thro } \\
\text { ughp } \\
\text { ut }\end{array}$ & Jitter & $\begin{array}{l}\text { Packet } \\
\text { Length }\end{array}$ & $\begin{array}{l}\text { Packet } \\
\text { Loss }\end{array}$ & $\begin{array}{l}\text { Throu } \\
\text { ghput }\end{array}$ & Jitter & $\begin{array}{l}\text { Packet } \\
\text { Length }\end{array}$ & $\begin{array}{l}\text { Packet } \\
\text { Loss }\end{array}$ & $\begin{array}{c}\text { Throug } \\
\text { hput }\end{array}$ & Jitter & $\begin{array}{l}\text { Packet } \\
\text { Length }\end{array}$ & $\begin{array}{c}\text { Packet } \\
\text { Loss }\end{array}$ \\
\hline RIP & 21 & 35.9 & 103 & 4 & 12 & 60.25 & 101 & 6 & 22 & 12.23 & 102 & 3 & 18.33 & 36.12 & 102.00 & 4.33 \\
\hline OSPF & 16 & 23.83 & 105 & 6 & 17 & 58.59 & 97 & 4 & 20 & 4.114 & 102 & 0 & 17.67 & 28.84 & 101.33 & 3.33 \\
\hline EIGRP & 26 & 10.14 & 78 & 3 & 26 & 55.13 & 86 & 3 & 32 & 7.0 & 102 & 2 & 28.00 & 24.09 & 88.66 & 2.66 \\
\hline RIP-OSPF & 19 & 2.519 & 98 & 5 & 17 & 31.9 & 83 & 5 & 12 & 15.57 & 104 & 7 & 16.00 & 16.66 & 95.00 & 5.66 \\
\hline RIP-EIGRP & 22 & 25.57 & 83 & 4 & 22 & 13.17 & 83 & 4 & 27 & 4.2 & 99 & 5 & 23.67 & 14.31 & 88.33 & 4.33 \\
\hline $\begin{array}{l}\text { OSPF- } \\
\text { EIGRP }\end{array}$ & 30 & 3.78 & 86 & 2 & 19 & 8.1 & 95 & 5 & 17 & 0.997 & 94 & 4 & 22.00 & 4.29 & 91.66 & 3.66 \\
\hline $\begin{array}{l}\text { RIP-OSPF- } \\
\text { EIGRP }\end{array}$ & 15 & 1.89 & 97 & 9 & 8 & 7.19 & 92 & 10 & 19 & 0.940 & 95 & 8 & 14.00 & 3.34 & 94.66 & 9.0 \\
\hline
\end{tabular}

There are several future research areas including (I) exploring other routing protocols and its combination with which we could further demonstrate the performance and utility of the network; (II) designing and implementing modern communication secured networks is inevitable for more scientific research in future to combat cybercrimes and network vulnerabilities where different types of cryptographic algorithms like encryption protocols, authentication and hashing protocols are utilized. Therefore, further research will be conducted also to evaluate the particular routing protocols performance in case of using different security protocols in the networks; (III) Our proposed protocols from this research paper will be further examined and validated for different other security- enabled networking environments.

\section{Conflict of Interest}

There is no conflict of interest for this research work.

\section{Acknowledgment}

This research work has been done in Advanced Networking Lab in Institute of Information and Communication Technology with the equipment supported by Bangladesh University of Engineering and Technology.

\section{References}

[1] K. Akunuri, R. Arora, and I. G. Guardiola, "A study of speed aware routing for mobile ad hoc networks" in International Journal of Interdisciplinary Telecommunications and Networking, Vol 3, pp. 40-61, 2011. https://dl.acm.org/citation.cfm?id=2440280

[2] S. Y. Jalali, S. Wani, M. Derwesh, "Qualitative Analysis and Performance Evaluation of RIP, IGRP, OSPF and EGRP Using OPNET” Research India Publications., Vol. 4, pp. 389-396, 2014. https://pdfs.semanticscholar.org/b616/f7b1a8e13f18b71998c557dc6f18d1f cba33.pdf

[3] M. Abdulkadhim, "Routing Protocols Convergence Activity and Protocols Related Traffic Simulation with Its Impact on the Network" in international Journal of Computer Science Engineering and Technology (IJCSET), vol. 5, no. $3, \quad$ pp. 40-43 (2015).http://ijcset.net/docs/Volumes/volume5issue3/ijcset2015050302.pdf

[4] C. Fancy, L. M. M. Thanveer,"An evaluation of alternative protocols-based Virtual Private LAN Service (VPLS)" in IoT and Application (ICIOT), International Conference, Nagapattinam, India, May. 2017, pp. 1-6 (2017) https://ieeexplore.iee. org/document/8073621/

[5] S.U. Masruroh, F. Robby, and N. Hakiem, "Performance Evaluation of Routing Protocols RIPng, OSPFv3, and EIGRP in an IPv6 Network" in International Conference on Informatics and Computing (ICIC), Mataram, Indonesia Oct. 2016, pp. 111-116 (2016). https://ieeexplore.ieee.org/document/7905699/

[6] D. R. Al-Ani, A. R. Al-Ani, "The performance of IPv4 and IPv6 in terms of Routing Protocols using GNS 3 Simulator" in 9th International Conference on Ambient Systems, Networks and Technologies, ANT-2018 and the 8th International Conference on Sustainable Energy Information Technology, SEIT 2018, May. 2018, pp. 1-6 (2018). https://dl.acm.org/citation.cfm?id=3223610 
[7] B. Swami,, and R. Singh,, "Performance analysis of DFS based ordered walk learning routing protocol in MANET" in Green Computing and Internet of Things (ICGCIoT), 2015 International Conference, Noida, India, Oct. 2015. https://ieeexplore.ieee.org/document/7380456/

[8] S. G. Thornier, "Dynamic routing protocol implementation decision between EIGRP, OSPF, and RIP based on technical background using OPNET Modeler" in Proc. Second International Conference on Computer and Network Technology (ICCNT), Bangkok, Thailand, Apr. 2010, pp. 191195. https://ieeexplore.ieee.org/document/5474509/

[9] X. Du, M. Guizani, Y. Xiao, H. H. Chen, "A Routing-Driven Elliptic Curve Cryptography based Key Management Scheme for Heterogeneous Sensor Networks" IEEE Transactions on Wireless Communications, vol. 8, no. 3, pp. 1223-1229, March 2009. https://ieeexplore.ieee.org/document/4801475/

[10] D. Xu, and L. Trajkovic, "Performance Analysis of RIP, EIGRP, and OSPF using OPNET" Simon Fraser University., British Columbia, Canada,2012. https://www.researchgate.net/publication/267385378_Performance_Analy sis_of_RIP_EIGRP_and_OSPF_using_OPNET

[11] E. Balasas, M. Roumeliotis, K.E. Psannis, "Performance Evaluation of Routing Protocols for BIG Data application," in University of Macedonia.,Thessaloniki, Greece, May,2018.

https://www.researchgate.net/publication/322291489_Performance_Evalua tion_of_Routing_Protocols_for_BIG_Data_application

[12] S. Corson and J. Macker. "Mobile Ad Hoc Networking (MANET): Routing Protocol Performance Issues and Evaluation Consideration" available at http://www.ietf.org/rfc/rfc2501.txt

[13] K. A. Zahoor, "A Novel Patient Monitoring Framework and Routing Protocols for Energy \& Qos Aware Communication in Body Area Network," $\mathrm{PhD}$ thesis, Dalhousie University, June 2013

[14] T. S. Andrew, W. J. David, Computer Networks, Prentice Hall, 2010.

[15] L. Chappell, Wireshark Network Analysis. Protocol Analysis Institute, San Jose, CA 95129 USA, 2012. 